Journal of Patient-Centered

\title{
Incidental Finding of Anomalous Pulmonary Venous Return of the Left Upper Lobe, a Rare Anatomical Variation
}

Anika Dutta

Loren Zuiderveld

Follow this and additional works at: https://aah.org/jpcrr

Part of the Cardiology Commons, Cardiovascular System Commons, Circulatory and Respiratory Physiology Commons, Diagnosis Commons, and the Radiology Commons

\section{Recommended Citation}

Dutta A, Zuiderveld L. Incidental finding of anomalous pulmonary venous return of the left upper lobe, a rare anatomical variation. J Patient Cent Res Rev. 2021;8:277-80. doi: 10.17294/2330-0698.1809

Published quarterly by Midwest-based health system Advocate Aurora Health and indexed in PubMed Central, the Journal of Patient-Centered Research and Reviews (JPCRR) is an open access, peer-reviewed medical journal focused on disseminating scholarly works devoted to improving patient-centered care practices, health outcomes, and the patient experience. 


\title{
Incidental Finding of Anomalous Pulmonary Venous Return of the Left Upper Lobe, a Rare Anatomical Variation
}

\author{
Anika Dutta, MD, ${ }^{1}$ Loren Zuiderveld, $\mathrm{MD}^{2}$ \\ ${ }^{1}$ Chicago Medical School, North Chicago, IL; ${ }^{2}$ Advocate Illinois Masonic Medical Center, Chicago, IL
}

\begin{abstract}
Partial anomalous pulmonary venous return (PAPVR) is a rare congenital abnormality in which 1 to 3 of the pulmonary veins connect to the right atrium rather than the left atrium. In this synthesis of the literature on PAPVR of the left upper lobe, we attempt to illustrate this clinical entity using a case detected incidentally on chest computed tomography, explain the anatomical aspects of this anomaly, and summarize the reported incidence and etiology of left-sided PAPVR. Lastly, differential diagnoses, clinical relevance, and management of left-sided PAPVR are presented. The identification of this variant is important, as it may have serious consequences. (J Patient Cent Res Rev. 2021;8:277-280.)
\end{abstract}

Keywords anatomy; embryology; CT imaging; differential; clinical presentation; management; partial anomalous pulmonary venous return

$\mathrm{P}$ artial anomalous pulmonary venous return (PAPVR) occurs when 1 to 3 of a person's pulmonary veins drain into the right atrium, either directly or through a systemic vein. ${ }^{1}$ PAPVR has a prevalence of $0.4 \%-0.7 \%$, and of these, only $10 \%$ are left-sided..$^{2}$ The most common presentation of PAPVR is a right upper lobe vein that drains into the right atrium. ${ }^{2}$ When PAPVR occurs in the left upper lobe, the left upper lobe pulmonary vein drains into a vertical vein that then drains into the brachiocephalic vein. ${ }^{3}$

The existing lack of knowledge on the subject of leftsided PAPVR was the impetus for conducting this brief review. Herein, we describe a classic clinical presentaton of left-sided PAPVR and summarize the scant literature on this entity.

\section{Illustrative Case}

A 61-year-old man with history of high cholesterol, impaired fasting glucose, and obesity presented to the emergency department with shortness of breath. He also reported chest pain described as throbbing in the left shoulder and intermittent retrosternal chest pain. His only prior thoracic imaging consisted of a coronary calcium screen study 16 months prior. A contrastenhanced computed tomographic (CT) chest pulmonary embolism protocol study was performed in the radiology department. The patient had a left upper lobe pulmonary

Corresponding author: Anika Dutta, MD,

Chicago Medical School, 3333 Green Bay Rd.,

North Chicago, IL 60064 (anika.dutta@my.rfums.org) vein draining into a vertical vein, which then drained into the brachiocephalic vein, indicative of left upper lobe PAPVR. Figures 1 and 2 display this anatomical structure as observed on CT.

The patient had no signs of acute process in the chest or pulmonary embolism. There was no evidence of cardiomegaly, pulmonary edema, or pulmonary arterial hypertension. There was also no evidence of other structural cardiac abnormalities such as atrial septal defect. However, the CT was a nongated study with significant motion artifact at the level of the atria, which precluded evaluation for atrial septal defect. The patient's shortness of breath and chest pain resolved in the emergency room and were classified as unspecified with no plan to undergo further workup as an outpatient. No further management was pursued for this incidental finding.

\section{Literature Search}

Using PubMed and Google Scholar, a review of the literature concerning PAPVR was conducted for articles written in the English language, with no restrictions placed on publication date or country. Search terms included: (anatomy, embryology, CT, imaging, differential, clinical presentation, OR management) AND partial anomalous pulmonary venous return. Additional articles were discovered by scanning the references of found papers as well as searching for articles that cited found papers. From this broad sample, articles that did not specifically discuss left-sided PAPVR were excluded from analysis.

\section{Search Results}

There is extensive literature on PAPVR in general but far less for left-sided. In all, we analyzed 9 articles that 


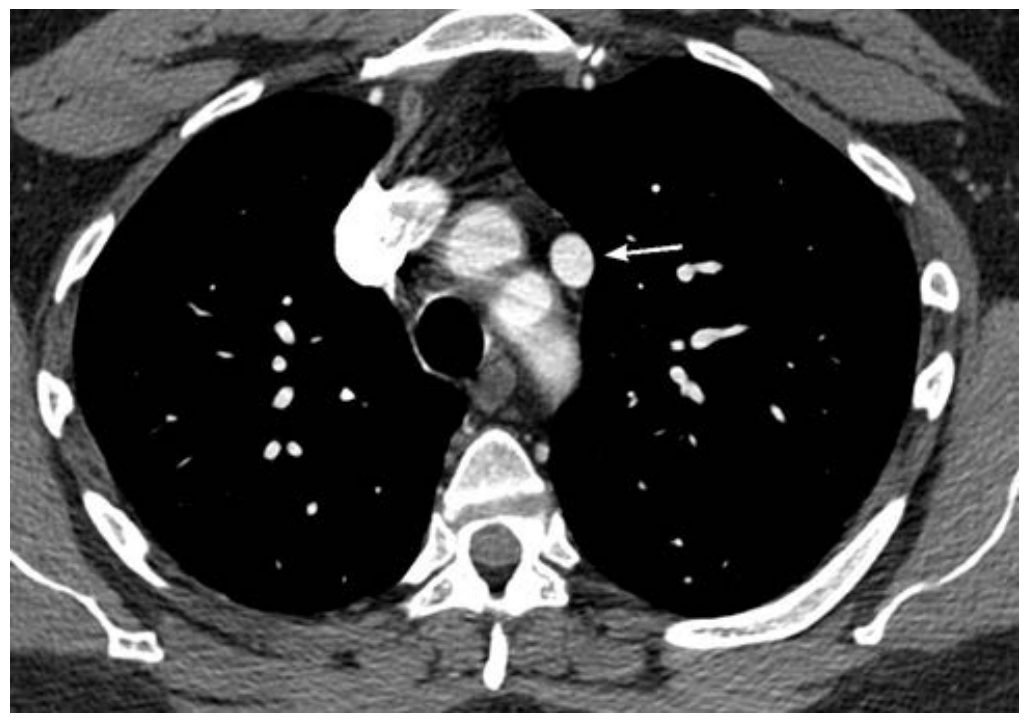

Figure 1. Axial computed tomographic scan of the chest demonstrating the vertical vein (arrow) along the left aspect of the superior mediastinum.

fulfilled the left-sided PAPVR review criteria. Of these, 6 discussed the rare left upper lobe type, as illustrated by our patient. Articles focused on the adult population, and 7 included case reports detailing a total of 8 patients (ranging in age from 33 to 68 years). The other 2 works were review-based and focused on CT findings or disease management. All of the articles used CT to assess and discuss pulmonary venous anatomy.

Of the 8 indiviudal patients described, 4 presented with symptoms such as cough, shortness of breath, or fatigue, with left-sided PAPVR identified on the subsequent chest CT. One patient was found to have left-sided PAPVR intraoperatively during a left upper lobectomy for non-small-cell carcinoma. Left-sided PAPVR was discovered in the remaining 3 patients due to imaging done for another indication, such as chest CT done on a lung cancer patient, wholebody CT taken to stage endometrial cancer, and CT done after insertion of a central venous line. Among the 8 patients presented in these case reports, leftsided PAPVR was managed nonsurgically in 5 . These patients were kept under regular follow-up to screen for indications for intervention, such as a significant shunt or right heart dilatation. The other 3 patients were managed surgically with lobectomy or heart-lung transplant if there were signs of right heart failure.

\section{Physiology}

Embryologically, PAPVR results from inadequate regression of primitive lung drainage. ${ }^{1}$ In the embryo, venous blood is drained from pulmonary vasculature into systemic veins. As the common pulmonary vein forms from the left atrium, it connects with the pulmonary vascular bed and the primitive connections with systemic veins disappear. This allows venous blood from the lung to drain into the common pulmonary vein and left atrium through 4 pulmonary veins. PAPVR arises when some of those pulmonary veins fail to connect with the common pulmonary vein, and part of the drainage to the systemic vein remains. ${ }^{1}$

Left-sided PAPVR presents with an atrial septal defect in $80 \%-90 \%$ of patients; $10 \%-15 \%$ of these are secundum type, and $85 \%$ are sinus venosus type. ${ }^{4}$ Left-sided PAPVR is occasionally associated with other cardiac abnormalities as well, including patent ductus arteriosus, pulmonic stenosis, and mitral stenosis. ${ }^{5}$

\section{Diagnosis}

Radiography in the setting of PAPVR is usually normal. ${ }^{3}$ The first-line modality in the diagnosis of PAPVR is CT, especially with iodinated contrast. ${ }^{6}$ It is useful in detecting the anomalous vein and its drainage as well as any associated lung abnormalities. However, the disadvantages of CT are the exposure to radiation and the possibility of impaired renal function due to intravenous iodinated contrast material. ${ }^{6}$ Alternatively, magnetic resonance imaging (MRI) may be used to evaluate the pulmonary veins. MRI is also helpful in detecting associated cardiac abnormalities such as atrial septal defect. ${ }^{6}$ Right heart size and left-to-right shunt, data important in directing treatment, can be quantified using gadolinium-enhanced magnetic resonance angiography. ${ }^{6}$

A differential diagnosis to left-sided PAPVR that should be considered by clinicians is a persistent left superior vena cava, as the two anomalies can have similar appearances 

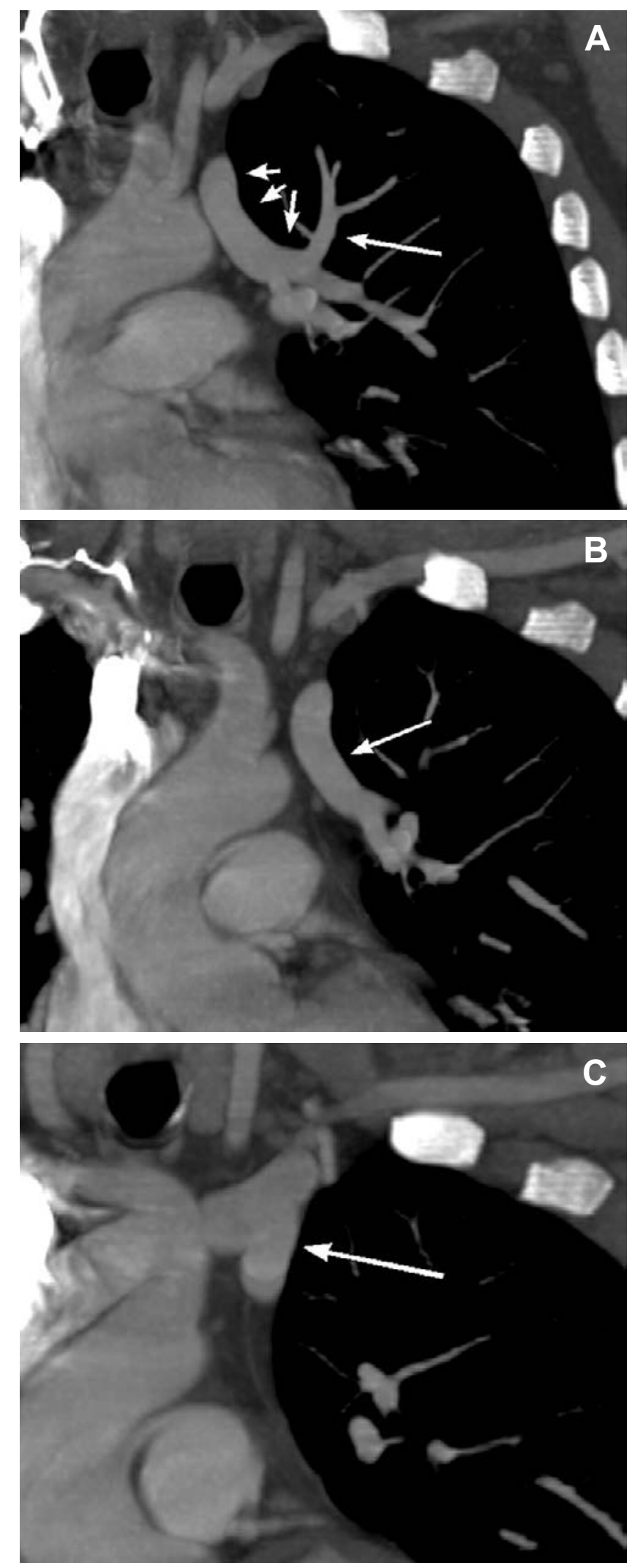

Figure 2. Coronal computed tomographic scan of the chest demonstrating the anomalous pulmonary vein rising from the left upper lobe of the lung $(\boldsymbol{A})$, draining into a vertical vein (B), and connecting to the left brachio-cephalic vein (C). on CT. Both are evidenced by a vertical anomalous vein lateral to the aortic arch. ${ }^{7}$ However, the number of vessels anterior to the left main bronchus can serve to aid in differentiating these anomalies. There is normally one vessel, the left superior pulmonary vein, anterior to the left main bronchus. In patients with duplication of the superior vena cava, two vessels are seen anterior to the left main bronchus, whereas no vessel is present in that location in patients with left-sided PAPVR. ${ }^{7}$ Another finding that differentiates the two anomalies is the course of the parahilar intraparenchymal veins in the left upper lobe. In duplication of the superior vena cava, they enter the left superior pulmonary vein anterior to the left main bronchus; in left-sided PAPVR, the veins enter the anomalous vessel at the level of the aorticopulmonary window. ${ }^{7}$ Furthermore, in duplication of the superior vena cava, the left innominate vein is most often absent or small, whereas it is normal or large in left-sided PAPVR. ${ }^{7}$

Another differential diagnosis is a pulmonary varix, which occurs with atresia or stenosis of one pulmonary vein resulting in localized dilatation of another. ${ }^{3}$ A pulmonary varix can be seen as tubular opacities that change in size with Valsalva maneuver on chest radiography, and as saccular dilatation of the vein on $\mathrm{CT}$ and MRI. ${ }^{3}$

A final differential is an enlarged left superior intercostal vein. ${ }^{3}$ On CT, this anomaly appears similar to the vertical vein seen in left-sided PAPVR, but it can be differentiated on radiography. On chest radiography, an "aortic nipple" measuring less than $4.5 \mathrm{~mm}$ may be seen at the convexity of the aortic arch. ${ }^{3}$

\section{Presentation and Management}

The clinical presentation of PAPVR of all types can vary from asymptomatic to congestive heart failure. Asymptomatic patients without signs of significant shunt may not need intervention; however they do require monitoring for signs of pulmonary arterial hypertension. ${ }^{6}$ Pulmonary arterial hypertension and increased pulmonary vascular resistance occur due to the left-to-right shunt that is formed by the persistent systemic venous connection and the resulting increase in pulmonary blood. ${ }^{1}$ This can cause right ventricular volume overload and eventually lead to tricuspid regurgitation, right atrial arrhythmias, and right ventricular failure. ${ }^{1}$ Presenting symptoms include dyspnea, fatigue, palpitations, and exercise intolerance. ${ }^{3}$

Management of left-sided PAPVR depends on severity of the disease. Although it is often an incidental finding, it can be clinically significant when $50 \%$ or more of the pulmonary blood flow returns through an anomalous connection. ${ }^{1}$ Surgery is indicated for symptomatic patients with a significant shunt, tricuspid regurgitation, 
or right ventricle enlargement. ${ }^{3}$ The surgical procedure involves anastomosing the vertical vein to the left atrial appendage, which is done via anterolateral thoracotomy. Possible complications of this procedure include cardiac arrest, atrial fibrillation, pulmonary venous obstruction, and complete heart block. ${ }^{3}$

Surgery is also indicated for some patients who will need a lobectomy for a primary lung neoplasm. ${ }^{8}$ If the neoplasm and PAPVR are located in different pulmonary lobes, PAPVR should be corrected during lobectomy, as a right heart failure may occur otherwise due to increased blood flow through the anomalous vein. If the neoplasm is in the same pulmonary lobe with PAPVR, the anomalous vessel may be resected during lobectomy. ${ }^{8}$ Lobectomy is the recommended treatment in cases of PAPVR where there is aplasia of 1 or more lobes of the right lung. ${ }^{9}$ This is called Scimitar syndrome, in which the anomalous venous return drains into the inferior vena cava and correction of the venous flow is therefore impossible. ${ }^{9}$

In patients with increased pulmonary vascular resistance and pulmonary arterial hypertension, the disease course is unlikely to be reversed with surgery and transplant may be the only option.

\section{Summary}

Partial anomalous pulmonary venous return is a rare anatomical anomaly, particularly in the left upper lobe. It is an important variant to recognize, as the clinical presentation can vary from asymptomatic to significant clinical consequences including pulmonary arterial hypertension, right-heart overload, tricuspid regurgitation, and right ventricular dysfunction.

\section{Patient-Friendly Recap}

- Partial anomalous pulmonary venous return of the left upper lobe is an even rarer form of the atypical anatomy known as PAPVR. In this "left-sided" PAPVR, one or more pulmonary veins drain into systemic blood vessels rather than the left atrium.

- The authors reviewed the limited literature available on left-sided PAPVR to offer a more comprehensive understanding of this antomical anomaly, which is often asymptomatic and discovered incidentally but may be a cause serious cardiovascular problems.

- Chest CT or MRI is used to map a person's veins and help rule out other possible diagnoses. While some patients with left-sided PAPVR can be only monitored for the rise of symptoms, those who require treatment usually undergo surgery.

\section{Author Contributions}

Study design: Zuiderveld. Data acquisition or analysis: Dutta, Zuiderveld. Manuscript drafting: Dutta. Critical revision: Zuiderveld.

\section{Conflicts of Interest}

None.

\section{References}

1. Sears EH, Aliotta JM, Klinger JR. Partial anomalous pulmonary venous return presenting with adult-onset pulmonary hypertension. Pulm Circ. 2012;2:250-5. CrossRef

2. Javangula K, Cole J, Cross M, Kay PH. An unusual manifestation of left partial anomalous pulmonary venous connection. Interact Cardiovasc Thorac Surg. 2010;11:846-8. CrossRef

3. Nath R, Murphy W, Aronson B. Rare case of left upper lobe partial anomalous pulmonary venous connec-tion. I Radiol Case Rep. 2013;7(6):9-14. CrossRef

4. Edwin F. Left-sided partial anomalous pulmonary venous connection - should diagnosis lead to surgery? Interact Cardiovasc Thorac Surg. 2010;11:847-8. CrossRef

5. Gaffey AC, Ball C, Katz S, Singhal S. Partial anomalous venous drainage of the left upper lobe during a robot-assisted pulmonary resection. $J$ Thorac Dis. 2016;8:E517-9. CrossRef

6. Tricarico E, Tricarico F, Florio C. Left-sided upper partial anomalous pulmonary venous return through a curved vein joining the left brachiocephalic vein. Case Rep Radiol. 2016;2016:1780909. CrossRef

7. Dillon EH, Camputaro C. Partial anomalous pulmonary venous drainage of the left upper lobe vs duplica-tion of the superior vena cava: distinction based on CT findings. AJR Am J Roentgenol. 1993;160:375-9. CrossRef

8. Liu Z, Yang R, Shao F, Pan Y. Right lung cancer with partial anomalous pulmonary venous return and ab-sent right upper lobe. J Thorac Dis. 2015;7:E186-8. CrossRef

9. Basalus MWZ, Said SAM, Stassen CM, Fast JH. Clinical and diagnostic features of partially anomalous pulmonary venous connection in an adult female patient: a case report and review of the literature. Neth Heart J. 2011;19:256-8. CrossRef

(C) 2021 Advocate Aurora Health, Inc. 